\title{
Nuevo índice pronóstico de mortalidad en ancianos luego de la internación
}

Development and Validation of a Prognostic Index for 1-year mortality in older adults after hospitalization. Walter LC, Brand RJ, Counsell SR et al. JAMA 2001;285:2987-2994.

\section{Objetivo}

Desarrollar y validar una regla de predicción clínica de mortalidad a un año en pacientes ancianos dados de alta hospitalaria.

\section{Diseño}

Datos derivados de dos estudios prospectivos con un año de seguimiento entre 1993 y 1997

\section{Lugar}

Estados Unidos.

\section{Pacientes}

El índice se derivó* de una cohorte de 1495 pacientes mayores de 70 años internados en una sala de clínica médica y se validó* en otra cohorte de 1427 pacientes en otro hospital diferente. Se incluyeron pacientes que fueron internados por una estadía mayor a 2 días.Se excluyeron las internaciones programadas, internaciones por subespecialidades o de cuidados intensivos.

\section{Evaluación de Factores Pronósticos}

Se tomaron datos registrados durante la internación. Se evaluaron características demográficas, actividades de la vida diaria (AVD), comorbilidades, estadía hospitalaria, y datos de laboratorio. Se calculó el puntaje de APACHE II y el de Charlson. Las variables a estudiar se seleccionaron según relevancia clínica, estudios predictores previos de mortalidad y prevalencia del $10 \%$ en la población.

\section{Medición de Resultados Principales}

Mortalidad a un año de seguimiento. Se obtuvo un $100 \%$ de seguimiento.

\section{Resultados Principales}

Se identificaron seis factores que independientemente se relacionaban con mortalidad: sexo masculino, número de AVD dependiente, insuficiencia cardíaca, cáncer, insuficiencia renal e hipoalbuminemia.

Las siguientes variables, al ajustar por estatus funcional, no se asociaron independientemente: edad, demencia y derivación a instituto geriátrico.

Se realizó un índice basado en un sistema aditivo de puntos, utilizando estos seis factores de riesgo y su relevancia relativa.

\begin{tabular}{l|c|c}
\hline FACTOR DE RIESGO & PUNTAJE & $\begin{array}{c}\text { Odds Ratio AJUSTADO } \\
\text { (IC 95\%) }\end{array}$ \\
\hline $\begin{array}{l}\text { Sexo Mascul ino } \\
\text { Actividades de la Vida Diaria (AVD) }\end{array}$ & 1 & $1,4(1,1-1,8)$ \\
- Dependiente en 1-4 AVD & 2 & $2,1(1,6-2,8)$ \\
- Dependiente en todas las AVD & 5 & $5,7(4,2-7,7)$ \\
\hline Comorbilidades & 2 & $2,0(1,5-2,5)$ \\
- Insuficiencia Cardiaca & 3 & $2,6(1,7-3,9)$ \\
- Cancer solitario & 8 & $13,4(6,2-29)$ \\
- Cancer metastásico & & \\
\hline Datos de laboratorio al ingreso & 2 & $1,7(1,2-2,5)$ \\
- Oreatinina > 3 mg/dl & 1 & $1,7(1,2-2,3)$ \\
- Albúmina 3,0-3,4 g/dl & 2 & $2,1(1,4-3,0)$ \\
- Albúmina <3,0 g/dl & &
\end{tabular}

La mortalidad al año en la cohorte de derivación* fue:

\begin{tabular}{|l|c|}
\hline Puntos & Mortalidad \\
\hline $0-1$ & $4 \%$ \\
$2-3$ & $19 \%$ \\
$4-6$ & $34 \%$ \\
$>6$ & $64 \%$ \\
\hline
\end{tabular}

El índice presentó una buena exactitud. El área bajo la curva ROC* en la cohorte de derivación fue de 0,75 y en la de validación de 0,79.

\section{Conclusiones}

El índice presentó buena discriminación, mejor que la de índices previos. El uso de este índice puede ser útil para el manejo de los pacientes y para el ajuste de riesgo de poblaciones de pacientes.

\section{Comentario}

La capacidad de predecir la evolución de los pacientes, de definir su pronóstico, es una de las funciones más esenciales y primitivas del rol del médico.

Por otro lado, el análisis cuantitativo del pronóstico y el uso de reglas de predicción concuerda con los ideales de la medicina basada en la evidencia. El usar información pronóstica ayuda a validar las impresiones clínicas y a corregir las creencias erróneas.Así mismo, dentro del contexto actual de la medicina gerenciada, los sistemas de ajuste de riesgo de poblaciones están tomando cada vez mayor importancia e interés académico.En la actualidad, existen programas comerciales para realizar el ajuste de riesgo basado en códigos de diagnóstico, así como varios índices predictores de mortalidad, en particular en pacientes hospitalizados. ${ }^{2}$

Esta nueva regla pronóstica se realizó tomando los datos de dos cohortes de estudios previos en pacientes que fueron hospitalizados y en seguimiento ambulatorio. Se generó una regla de fácil uso cotidiano basada en seis factores a los cuales se les otorgó un puntaje ponderado.

Esta regla abarca múltiples áreas clínicas. Esta multidimensionalidad se asocia a una mayor exactitud pronóstica que los otros índices evaluados. Mientras que el índice generado presentó área bajo la curva $\mathrm{ROC}$ de 0,79 , el correspondiente al índice de comorbilidades de Charlson fue de 0,68 y el del índice de orientación fisiopatológica APACHE II de 0,59.

Por otro lado, este estudio demuestra la importancia pronóstica del estatus funcional en pacientes ancianos. Es decir, al ajustar por estatus funcional (AVD) muchas variables como la edad y diferentes enfermedades, tales como demencia, no tuvieron valor pronóstico. En este sentido, el estatus funcional es la expresión final, la resultante de la repercusión clínica de múltiples enfermedades (típica situación en ancianos) y el reflejo de la severidad de las mismas. Como limitante de este estudio las variables estudiadas estaban limitadas a los datos registrados en los estudios previos, los cuales no tenían como objetivo primario el desarrollo de un índice pronóstico. Por último, sería interesante evaluar este índice pronóstico en poblaciones de pacientes ancianos de nuestro medio.

Conclusiones del comentador: este nuevo índice que incluye seis factores de riesgo parece superior a los previos y destaca la importancia pronóstica del etatus funcional en ancianos.

\section{Dr. Juan L Bernabó [ Servicio Clínica Médica.Plan de Salud.Hospital Italiano de Buenos Aires. ]}

\section{Referencias}

1.Chirstakis NA.Death Foretold:Prophecy and Prognosis in Medical Care.Chicago.Universtiy of Chicago Press.1999.

2.Desai MM, Bogardus ST Jr, Williams CS et al.Development and validation of a risk-adjustment index for older patients:the high-risk diagnoses for the elderly scale. J Am Geriatr Soc $2002 \mathrm{Mar} ; 50(3): 474-81$ 